\title{
A STIELTJES INTEGRAL EQUATION ${ }^{1}$
}

F. G. DRESSEL

Introduction. By making use of the notion of derivative taken with respect to a function of bounded variation, solutions of the YoungStieltjes integral equations of the following type

$$
F(x)=M(x)+\lambda \int_{0}^{1} H(x, y) d F(y)
$$

are obtained in this paper. Since the integration by parts formula is not valid for Young-Stieltjes integrals, equation (1) cannot be immediately changed to the equation

$$
f(x)=m(x)+\lambda \int_{0}^{1} f(y) d K(x, y) .
$$

Fischer ${ }^{2}$ in his treatment of equation (2) imposed three conditions on $K(x, y)$ to obtain solutions. In the second part of this paper, we show that one of these conditions is sufficient to insure the existence of a bounded monotone increasing function $g(y)$ such that

$$
\left|K\left(x, y_{2}\right)-K\left(x, y_{1}\right)\right| \leqq\left|g\left(y_{2}\right)-g\left(y_{1}\right)\right| .
$$

It has been shown ${ }^{3}$ that if condition (3) holds, then equation (2) can be changed into an ordinary Fredholm equation. Thus it appears that only one of the three conditions imposed on $K(x, y)$ by Fischer is needed to insure the solution of (2).

Before handling equation (1), let us note that if $g\left(y_{1}\right)<g\left(y_{2}\right)$ for $y_{1}<y_{2}$, we may apply the transformation ${ }^{4}$

$$
\beta(s)=\lim \sup E_{y}[s \geqq g(y)], \quad g(0) \leqq s \leqq g(1),
$$

and send the integral equation

$$
v(x)=w(x)+\lambda \int_{0}^{1} G(x, y) v(y) d g(y)
$$

in to the Fredholm equation

${ }^{1}$ Presented to the Society, February 24, 1940.

2 C. A. Fischer, The Fredholm theory of the Stieltjes equation, Annals of Mathematics, (2), vol. 25 (1923-1924), pp. 124-158.

All functions used in this paper are assumed to be measurable Borel.

${ }^{3}$ F. G. Dressel, $A$ note on Fredholm-Stieltjes integral equations, this Bulletin, vol. 44 (1938), pp. 434-437.

${ }^{4} E_{y}[s \geqq g(y)]$ designates the set of values of $y$ such that $s \geqq g(y)$. 


$$
v(\beta(t))=w(\beta(t))+\lambda \int_{\alpha}^{\delta} G(\beta(t), \beta(s)) v(\beta(s)) d s,
$$

where $\alpha=g(0), \delta=g(1)$, and $t$ is any of the possible solutions of

$$
x=\beta(t), \quad g(0) \leqq t \leqq g(1) .
$$

Now in $L^{2}$ the Fredholm theory applies to (6) if

$$
\int_{0}^{r} \int_{0}^{1}|G(x, y)|^{2} d g(x) d g(y)=\int_{\alpha}^{\delta} \int_{\alpha}^{\delta}|G(\beta(t), \beta(s))|^{2} d t d s
$$

exists. ${ }^{5}$ Also if $h(t) \subset L^{2}$, we have by Schwartz's inequality

$$
\left|\int_{0}^{1} G(x, \beta(t)) h(t) d t\right|^{2} \leqq \int_{0}^{1}|G(x, \beta(t))|^{2} d t \int_{0}^{1}|h(t)|^{2} d t,
$$

and from this we see $\int_{0}^{1} G(x, \beta(t)) h(t) d t$ is integrable with respect to $g(x)$ if $(7)$ holds. The above statements lead to the following lemma:

LEMмa 1. If $g\left(x_{1}\right)<g\left(x_{2}\right)$ for $x_{1}<x_{2}$ with $g(x)$ bounded, and if under the transformation (4) the functions $w(x), G(x, y) \subset L^{2}$, and also if (7) holds, then for all values of $\lambda$, except perhaps the characteristic values ${ }^{5}$ of the integral equation (6), the equation (5) has a solution integrable with respect to $g(x)$.

Stieltjes integral equations. Consider now the equation (1) where the given functions $M(x)$ and $H(x, y)$ are assumed to be absolutely continuous $^{6} g(x)$. Here $g(x)$ is a bounded increasing function, continuous from the right except perhaps at $x=0$. Let $m(x)$ and $h(x, y)$ be the derived numbers of $M(x)$ and $H(x, y)$ taken with respect to $g(x)$, and form the equation

$$
v(x)=m(x)+\lambda \int_{0}^{1} h(x, y) v(y) d g(y) .
$$

We see from our lemma that equation (8) will have a solution under

\footnotetext{
${ }^{5}$ For list of references and terminology see E. Hille and J. D. Tamarkin, On the characteristic values of linear integral equations, Proceedings of the National Academy of Sciences, vol. 14 (1928), pp. 911-914.

${ }^{6}$ For terminology see A. J. Maria, Generalized derivatives, Annals of Mathematics, (2), vol. 28 (1926-1927), pp. 419-432. Let $f(x)$ be a function of bounded variation and denote by $\bar{f}(e)$ the completely additive function of sets defined by $f(x)$. (The facts here are that for any interval $\alpha\left(x_{1} \leqq t \leqq x_{2}\right)$ the relation $\bar{f}(\alpha)=f\left(x_{2}+0\right)-f\left(x_{1}-0\right)$ holds.) The function $f(x)$ is said to be absolutely continuous with respect to the non-decreasing function $g(x)$ if whenever $E$ is a set for which $\bar{f}(E)$ is defined and $\bar{g}(E)=0$, then $\bar{f}(E)=0$.
} 
rather weak restrictions on $m(x)$ and $h(x, y)$. Let us now assume (8) has a solution, say $\bar{v}(x)$, which is integrable $g(x)$, and define the function $Q(y)$ as

$$
\begin{aligned}
Q(y) & =\int_{0}^{y} \bar{v}(x) d g(x), & & y \neq 0, \\
& =0, & & y=0 .
\end{aligned}
$$

We are now able to write the solution of (1) as

$$
F(x)=M(x)+\lambda \int_{0}^{1} H(x, y) d Q(y) .
$$

To see that the above $F(x)$ furnishes a solution of (1), substitute the right side of (9) in both sides of equation (1). After cancelling $M(x)$ we have

$$
\begin{aligned}
\lambda \int_{0}^{1} H(x, y) d Q(y)= & \lambda \int_{0}^{1} H(x, y) d M(y) \\
& +\lambda^{2} \int_{0}^{1} H(x, y) d_{y} \int_{0}^{1} H(y, t) d Q(t) .
\end{aligned}
$$

Since $\bar{v}(x)$ is a solution of (8), on integration of that equation with respect to $g(x)$, there results

$$
\begin{array}{rlrl}
Q(x) & =M(x+0)-M(0)+\lambda \int_{0}^{1}[H(x+0, t)-H(0, t)] \bar{v}(t) d g(t),{ }^{7} x \neq 0, \\
& =M(x+0)-M(0)+\lambda \int_{0}^{\alpha}[H(x+0, t)-H(0, t)] d Q(t), & x \neq 0, \\
& =0, & x=0 .
\end{array}
$$

If the above is substituted in the left side of (10), it is easily seen to be equal to the right side, hence (9) satisfies (1).

From the above and our lemma we have the following theorem:

THEOREM 1. Let $g(x)$ be continuous from the right in $0<x \leqq 1$, and satisfy the conditions of Lemma 1 ; let $M(x)$ and $H(x, y)$ in equation (1) be absolutely continuous $g(x)$ and have $m(x)$ and $h(x, y)$ as derived numbers $(g(x))$. Then if $m(x), h(x, y)$ satisfy the conditions placed on $w(x)$, $G(x, y)$ respectively in Lemma 1, equation (9) furnishes a solution of (1) for all values of $\lambda$ except those that are characteristic values of the integral equation (8).

\footnotetext{
${ }^{7}$ F. G. Dressel, loc. cit., p. 435.
} 
Corollary. If

$$
\begin{gathered}
\left|H\left(x_{2}, y\right)-H\left(x_{1}, y\right)\right| \leqq\left|g\left(x_{2}\right)-g\left(x_{1}\right)\right|, \\
\left|M\left(x_{2}\right)-M\left(x_{1}\right)\right| \leqq\left|g\left(x_{2}\right)-g\left(x_{1}\right)\right|
\end{gathered}
$$

where $g(x)$ meets the requirements of Theorem 1 , then (9) is the solution of (1) for all values of $\lambda$ that are not characteristic values of the integral equation (8).

The Fischer theory. In the treatment of equation (2), Fischer imposed the following condition on the kernel $K(x, y)=K(x, y+0)$ :

$$
V_{2} K(x, y)<N \text {. }
$$

Here $N$ is a constant and $V_{2} K$ has the following meaning:

$$
V_{2} K(x, y)=\text { l.u.b. } \sum_{i=1}^{n}\left|K\left(x_{i}, y_{i}\right)-K\left(x_{i}, y_{i-1}\right)\right|,
$$

it being understood that $0=y_{0}<y_{1}<\cdots<y_{n}=1$, and $\left\{x_{i}\right\}$ are any $x$ 's in $0 \leqq x \leqq 1$. We shall soon show that the above condition implies that inequality (3) holds.

Define the set $E$ on the interval $0 \leqq y \leqq 1$ to be given by

$$
y=m / 2^{n}, \quad m=0,1,2, \cdots, m \leqq 2^{n}, n=1,2, \cdots,
$$

and let $g(x, y)$ be a function with the properties

$$
g(x, y)=g(y, x) \geqq 0, \quad g(x, y) \leqq g(x, z)+g(z, y)
$$

and if $0 \leqq y_{0}<y_{1}<\cdots<y_{n} \leqq 1$ then

$$
\sum_{i=1}^{n} g\left(y_{i}, y_{i-1}\right)<N
$$

for all $n$.

Let $y_{j} \subset E$ and define $A_{n}\left(y_{j}\right)$ as

$$
\begin{aligned}
A_{n}\left(y_{j}\right) & =\sum_{i=1}^{m} g\left(\frac{i}{2^{n}}, \frac{i-1}{2^{n}}\right), & 0<\frac{m}{2^{n}} \leqq y_{j}<\frac{m+1}{2^{n}}, \\
& =0, & y_{i}<\frac{1}{2^{n}} .
\end{aligned}
$$

Due to the conditions on $g(x, y)$ we have

$$
A_{n}\left(y_{j}\right) \leqq A_{n+1}\left(y_{j}\right), \quad A_{n}\left(y_{j}\right)<N ;
$$

hence $\lim _{n \rightarrow \infty} A_{n}\left(y_{j}\right)$ exists: let us call it $A\left(y_{j}\right)$. Again let $y_{1}, y_{2} \subset E$ with $y_{1}<y_{2}$; then if $y_{1}=r / 2^{k}, y_{2}=m / 2^{k}$, 


$$
A_{k}\left(y_{2}\right)-A_{k}\left(y_{1}\right)=\sum_{i=r+1}^{m} g\left(\frac{i}{2^{k}}, \frac{i-1}{2^{k}}\right) \geqq g\left(y_{2}, y_{1}\right) ;
$$

hence

$$
A_{k}\left(y_{2}\right)-A_{k}\left(y_{1}\right) \geqq g\left(y_{2}, y_{1}\right) \geqq 0 .
$$

Thus $A(y)$ as defined on $E$ is a bounded monotone increasing function; hence we may extend the definition of $A(y)$ on $C E$ so as to define a monotone increasing function of bounded variation ${ }^{8}$ on $0 \leqq y \leqq 1$. We make $A(y)$ unique by taking $A(y+0)=A(y)$ if $y \subset C E$.

Turn now to Fischer's condition $V_{2} K(x, y)$ and define

$$
g\left(y_{2}, y_{1}\right)=\underset{0 \leqq x \leqq 1}{\text { l.u.b. }}\left|K\left(x, y_{2}\right)-K\left(x, y_{1}\right)\right| \text {. }
$$

This $g(x, y)$ is easily seen to satisfy conditions (a) and (b); hence the following theorem is valid.

THEOREM 2. If for the function $K(x, y)=K(x, y+0)$ the condition $V_{2} K(x, y)<N$ holds, then there exists a bounded monotone increasing function $A(y)$ such that

$$
\left|K\left(x, y_{2}\right)-K\left(x, y_{1}\right)\right| \leqq\left|A\left(y_{2}\right)-A\left(y_{1}\right)\right| \text {. }
$$

Corollary. The condition $V_{2} K(x, y)<N$ imposed on $K(x, y)$ $=K(x, y+0)$ by Fischer is sufficient to change equation (2) into an ordinary Fredholm equation. ${ }^{9}$

The existence of the following limit is essential in Fischer's treatment of equation (2):

$$
\lim _{\delta \rightarrow 0} \sum_{i=1}^{n}\left[K\left(x_{i}, x_{i}\right)-K\left(x_{i}, x_{i-1}\right)\right], \quad 0=x_{0}<x_{1}<\cdots<x_{n}=1,
$$

where $\delta=\max \left(x_{i}-x_{i-1}\right)$. The Fischer theory will therefore not apply to the kernel

$$
\begin{aligned}
K_{1}(x, y) & =1, & & x=\frac{1}{2}, \\
& =0, & & \text { elsewhere. }
\end{aligned}
$$

However, the integral equation (2) with the above kernel is easily treated since $V_{2} K_{1}(x, y)<2$.

BROWN UNIVERSITY AND DUKe UNIVERSITy

${ }^{8}$ G. C. Evans, Logarithmic Potential, American Mathematical Society Colloquium Publications, vol. 6, 1927, pp. 5-6.

${ }^{\circ}$ F. G. Dressel, loc. cit., p. 436. 